Témoigner Témoigner. Entre histoire et mémoire

Getuigen Revue pluridisciplinaire de la Fondation Auschwitz

$115 \mid 2013$

L'Espagne en construction mémorielle

\title{
Fosses communes, paysages de la terreur
}

Mass graves, landscapes of terror

Massagraven, landschappen van terreur

\section{Francisco Ferrándiz}

Traducteur : Nadia Tahir

\section{(2) OpenEdition}

Journals

\section{Édition électronique}

URL : http://journals.openedition.org/temoigner/476

DOI : 10.4000/temoigner.476

ISSN : 2506-6390

Éditeur :

Éditions du Centre d'études et de documentation Mémoire d'Auschwitz, Éditions Kimé

\section{Édition imprimée}

Date de publication : 1 mars 2013

Pagination : 44-67

ISBN : 978-2-84174-628-6

ISSN : 2031-4183

Référence électronique

Francisco Ferrándiz, «Fosses communes, paysages de la terreur », Témoigner. Entre histoire et mémoire [En ligne], 115 | 2013, mis en ligne le 01 juin 2015, consulté le 23 octobre 2020. URL : http:// journals.openedition.org/temoigner/476 ; DOI : https://doi.org/10.4000/temoigner.476 


\title{
Fosses communes, paysages de la terreur*
}

\author{
FRANCISCO FERRÁNDIZ \\ Instituto de Lengua, Literatura y Antropología (ILLA) du \\ Centro de Ciencias Humanas y Sociales (CCHS) del Consejo \\ Superior de Investigaciones Científicas (CSIC), Madrid
}

Pour Chun, pour son courage exquis

\section{SPASMES JUDICIAIRES ET MÉDIATIQUES}

Le 30 octobre 2008, ont commencé les opérations de recherche des corps de cinq femmes, dans le lieu connu sous le nom de «Las Albarizas » dans le village de Tudal, municipalité de Villanueva de la Vera à Caceres. Florentina Quintana Huertas, ses deux filles Ángela et Ana Tornero Quintana, Úrsula Sánchez Mate et Bernarda García Hernández ont été assassinées le 26 septembre 1936 par un groupe de dix phalangistes de leur village. L'excavation des deux lieux dans lesquels les témoignages oraux situaient leurs tombes s'est produite au milieu d'une tourmente judiciaire et médiatique. Cette dernière a été déclenchée par la décision du juge Baltasar Garzón

\footnotetext{
(*) Ce texte se base sur un travail de terrain «multi-situé » (George E. Marcus, «Ethnography in/of the World System: The Emergence of Multi-Sited Ethnography », in Annual Review of Anthropology, n² 24, 1995, p. 95 117) de plus de cinq ans sur le cycle le plus récent d'exhumations de fosses communes de la guerre civile espagnole. Il a été mené à bien dans plusieurs cadres, notamment des actes commémoratifs, des conférences sur la mémoire, des présentations de livres, des expositions artistiques, des exhumations (Valdediós, 2003 ; Villamayor, 2004 ; Fontanosas, 2006 ; Villanueva de la Vera, 2008, entre autres), des réinhumations, plusieurs ONG de « récupération de la mémoire historique » (surtout ARMH et « Nuestra Memoria »), des groupes de travail bénéficiant d'un soutien institutionnel (Sociedad de Ciencias Aranzadi) et des laboratoires de médecine légiste. Cette recherche comprend aussi une compilation systématique d'articles de presse, d'informations et de documentaires télévisuels et un travail de terrain sur internet où circulent de nombreuses informations (ainsi que beaucoup de désinformation) sur les différents aspects de la récupération de la mémoire historique de la guerre civile. On y trouve aussi de nombreux débats sur les exhumations et les circonstances dans lesquelles elles se déroulent. Je remercie très sincèrement Lucio García Tornero, Luis Ragel, Marisa Hoyos et Jimi Jiménez pour leur amabilité pendant l'exhumation de Villanueva de la Vera, et à nouveau Lucio García Tornero pour sa patience lors de mes nombreux appels téléphoniques pendant la rédaction de cet article. Je souhaiterais aussi remercier Luis Diaz, María García, Julián Lopez, Pedro Tomé et Javier Rodrigo pour leurs suggestions. Enfin, je remercie María García, pour mavoir prêté sa copie du Semanario Gráfico Nacional Sindicalista du 25 novembre 1939. Cette recherche a été menée à bien grâce au Proyecto Intramural Espacial (PIE) 2007101006 du CSIC.
} 
de se déclarer compétent pour juger les crimes du franquisme dans une ordonnance controversée signée le 16 octobre, moins de deux semaines plus tôt. À l'Audience nationale, la plus haute cour de justice espagnole, le juge Santiago Pedraz a autorisé cette excavation seulement quelques jours avant que la terre de Las Albarizas ne commence à être remuée. L'excavation a eu lieu parallèlement à d'autres, notamment celle de la « caisse collective ou columbarium » 198 du Valle de los Caídos, où se trouvent les corps de sept habitants du village Pajares de Adaja de la région d'Ávila. Ils ont été fusillés le 20 août 1936 par les troupes franquistes, exhumés par la suite et transférés en 1959 dans le cadre de l'opération rocambolesque, à échelle nationale, de captation de cadavres pour le mausolée de Franco, actuellement controversé1. Il s'agit de la même décision judiciaire. L'objectif était d'octroyer les conditions de « confidentialité et de dignité des victimes et de leurs familles » pour l'exhumation de la fosse susceptible de contenir les restes de Francisco Galadi, Dióscoro Galindo, Joaquín Arcollas et Federico García Lorca, sollicitée par la famille de ce dernier.

Cet itinéraire judiciaire a commencé le 14 décembre 2006, lorsque diverses associations ont introduit une requête devant la Cour centrale d'instruction numéro 5 de l'Audience nationale, dont le responsable est Baltasar Garzón. La requête demandait qu'il se déclare compétent pour enquêter et juger des délits présumés de détention illégale

fondamentalementà cause del'existence d'un plan systématique et préconçu d'élimination d'opposants politiques. La mise en place de celui-ci s'est faite à travers de multiples morts, tortures, exils et disparitions forcées ${ }^{2}$ (détentions illégales) de personnes à partir de 1936, pendant les années de la guerre civile et celles de l'après-guerre, qui ont eu lieu dans différents points du territoire espagnol ${ }^{3}$.

(1) On autorisait aussi l'exhumation de la caisse individuelle 10 672, située au Piso 1, Crypte África de la Basilique de la Santa Cruz du Valle de los Caídos, qui contient les restes de Fidel Canales, transférés le 30 décembre 1968 du cimetière de Griñón au Valle de los Caídos, où il se trouvait depuis son décès au front. Grâce à la recherche constante du corps de son père, de ceux de six voisins de Pajares de Adaja et de celui de son oncle Fidel, Fausta Canales contribue à révéler les aspects les plus sinistres de cette monumentale fosse commune. Je remercie Fausta pour son amabilité constante. Pour plus de détails, cf. Daniel Sueiro, El Valle de los Caídos: Los secretos de la cripta franquista [1976], Madrid, La Esfera de los Libros, 2006 ; Paloma Aguilar, Memoria y olvido de la Guerra Civil española, Madrid, Alianza, 1996, p. 116-129 et Queralt Solé i Barjau, Els morts clandestins: Les fosses comunes de la Guerra Civil a Catalunya (1936-1939). Barcelona, Editorial Afers, 2008, ainsi que le documentaire Franco: Operación Caídos, produit par Mediapro, présenté par Francisco González («Gonzo »), et diffusé sur la chaîne espagnole Antena 3 le 28 janvier 2009.

(2) Dans le contexte espagnol l'application croissante de la dénomination « disparu » (desaparecido) pour faire référence aux personnes fusillées pendant la Guerre civile et l'après-guerre (pour le moment surtout dans le camp des vaincus), a pris de l'ampleur avec l'impact public des démarches de Baltasar Garzón. Cette dénomination a ouvert une controverse (parfois vive) aux conséquences judiciaires, sociales, politiques, médiatiques et historiographiques, qui sera analysée dans un prochain article. Dans un cadre comparatiste, pour une analyse critique de la figure du « détenu-disparu », comme « catastrophe de l'identité » en Argentine et en Uruguay, cf. Gabriel Gatti, El detenido-desaparecido: Narrativas posibles para una catástrofe de la identidad, Montevideo, Trilce, 2008.

(3) Cf. l'ordonnance de Garzón du 16 octobre 2008, p. 3. 
Plus tard, d'autres initiatives privées et associations se sont engagées dans ce processus. Le choix du tribunal de Garzón pour présenter ces dénonciations n'était pas anodin. Cela faisait partie d'une stratégie qui souhaitait capitaliser son prestige international, consolidé par son intervention dans le « cas Pinochet », et sa visibilité médiatique avec l'espoir que les requêtes ne fussent pas, au minimum, classées sans suite. Ce déplacement de certaines associations vers la judiciarisation du processus de récupération de la mémoire historique partait aussi d'une insatisfaction répandue au sein de ces collectifs. En effet, elles estimaient être confrontées à des propositions timides et des avancées lentes, telles que la « Loi 52/2007 » du 27 décembre 2007 par laquelle : « des droits sont reconnus et étendus [, loi] qui établit des mesures en faveur de ceux qui ont souffert des persécutions ou de la violence pendant la guerre civile et le franquisme. » Elle est plus connue sous le nom de Loi sur la mémoire historique ${ }^{4}$

Pour saisir la sensation de vertige à laquelle nous avons déjà fait référence, je vais d'abord revenir brièvement sur certains des événements les plus marquants de ces derniers mois. Le 29 janvier 2008, le procureur général de l'État publie un rapport dans lequel il s'oppose à l'acceptation de la procédure des dénonciations présentées jusqu’à présent et recommande qu'elles soient classées. Le 23 juin, Garzón demande au Ministère de l'Intérieur et à celui de la Défense de lui envoyer les rapports qui portent sur des disparus, pour pouvoir prouver qu'aucune enquête politique ni judiciaire n'est menée sur les faits. Par ailleurs, il demande aux accusateurs une liste complète des données qu'ils possèdent. C'est alors qu'au sein des collectifs de récupération de la mémoire, se répand le sentiment que Garzón va s’impliquer, même s'ils ignorent les dimensions et le parcours juridique qui commence à se dessiner. Le $1^{\text {er }}$ septembre, Garzón demande à l'Abbé du Valle de los Caídos, aux Archives générales de la Nation, à la Conférence épiscopale, au Centre de documentation de la mémoire historique et à certaines mairies de lui fournir toutes les informations en leur possession sur les disparus. Le lendemain, il exige des associations qui se sont portées partie civile que, dans les quinze jours, elles remettent la liste des disparus et l'emplacement des fosses communes dans lesquelles ces derniers auraient pu être enterrés. Le 22 septembre, des représentants de la jeune Plateforme de victimes de disparitions forcées du franquisme (PVDFF) donnent à Garzón une liste comportant 143353 noms et organisent une conférence de presse à l'Ateneo de Madrid pour expliquer leur position.

Dans une ordonnance, le 16 octobre 2008, le juge Baltasar Garzón se déclare compétent pour prendre en charge la procédure pour délits permanents présumés de détention illégale dans le cadre de crimes contre l'humanité, pour un total de 114266 cas entre le 17 juillet 1936 et décembre 1951. Une tornade à la fois très intense et très brève - elle dure moins de quatre semaines - se produit alors. Des questions judiciaires techniques, des controverses politiques et un important remue-ménage médiatique se sont entrecroisés non seulement en Espagne, mais aussi à l'étranger. Dans ce document, perçu par le quotidien El Mundo dans son éditorial du lendemain

(4) Cf. http://www.boe.es/boe/dias/2007/12/27/pdfs/A53410-53416.pdf 
comme une «truculente garzonerie », le juge considère que pendant la guerre civile et le franquisme de graves violations de droits comparables à la catégorie juridique de crimes de lèse-humanité avaient eu lieu. Pour lui, le processus de disparitions forcées a été utilisé systématiquement pour empêcher l'identification des victimes et entraver l'action de la justice jusqu'à aujourd'hui. Parallèlement, il autorisait dixneuf excavations, sollicitées par les associations et des particuliers plaignants, parmi lesquelles s'est distinguée, pendant quelques jours, celle qui permettait d'obtenir les restes de Dióscoro Galindo González, Francisco Galadi Melgar, Joaquín Arcollas Cabezas et Federico García Lorca. Ces hommes étaient enterrés dans une fosse commune impliquée dans une importante controverse depuis des années. Le lendemain, dans une nouvelle ordonnance, Garzón transforme les démarches préalables en instruction ordinaire pour rendre la tâche plus difficile au recours prévu par le procureur. Le recours d'appel devant la Chambre pénale arrive le 20 octobre, signé par le procureur en chef Javier Zaragoza, qui dénonce l'« échafaudage juridique singulier » mis en place par Garzón dans son ordonnance pour justifier l'application du droit pénal ${ }^{5}$. Zaragoza estime qu'il s'agit de délits pour lesquels « il y a prescription ${ }^{6}$ » et qui ont été « amnistiés ${ }^{7}$ »et non pas des crimes contre l'humanité ${ }^{8}$. Selon le procureur, puisque la «justice des sanctions, qui est la quintessence de la procédure pénale » est écartée, les faits entreraient dans le cadre de réparations morales aux victimes, définies sous les termes de vérité, reconnaissance et réparation. Ils peuvent donc être interprétés sous la couverture de la « justice transitionnelle et réparatrice », largement couverts par la Loi sur la mémoire historique?.

Le 29 octobre, comme nous l'avons vu, le juge Pedraz, en remplacement de Garzón, prononce une décision qui autorise plusieurs exhumations parmi lesquelles se trouvent, dans un premier temps, celle de Villanueva de la Vera. Le 7 novembre, la Chambre pénale, en réunion extraordinaire, décide d'arrêter les exhumations autorisées dans le cadre de la procédure ouverte par Garzón jusqu’a ce que sa compétence pour le cas soit mieux définie. Le 18 novembre, Garzón en personne prononce une nouvelle décision établissant que le cas n'est plus de sa compétence. Il se dessaisit ainsi du dossier et l'oriente vers les tribunaux d'instance territoriale compétents. Il insiste sur le fait que son dessaisissement ne signifie pas que ces délits « ne sont plus » et qu'ils continuent d'être des crimes contre l'humanité. Dans ce contexte, il signe un surprenant texte à propos de ceux qu'on appelle « les enfants perdus du franquisme ». Il fait référence au présumé «montage pseudojuridique qui, supposément, a permis

(5) Cf. Javier Zaragoza, recours d'appel devant la chambre pénale, 20 octobre 2008, p. 3.

(6) Ibid., p. 22-31.

(7) Ibid., p. 31-35.

(8) Zaragoza écrit dans son recours : « la qualification juridique des faits dénoncés comme crimes de lèsehumanité n'est pas applicable dans ce cas, puisque le corps normatif écrit et coutumier qui constitue la légalité pénale internationale n'existait pas au moment des faits. Par ailleurs, cette qualification juridique ne peut être appliquée rétroactivement », p. 21.

(9) Ibid., p. 5-6. 
de couvrir l'enlèvement systématique d'enfants. » Ces enfants de mères et de familles républicaines devaient être élevés dans le cadre des préceptes du régime entre 1937 et 1950 (impliquant également des changements d'identité) et seraient pour la plupart vivants $^{10}$. Le 26 novembre, la Cour suprême publie une décision en réponse à la demande de la petite-fille de Juan Negrín, et dans laquelle elle déclare qu'il n'existe aucune raison ou base légale pour que cette instance judiciaire reconnaisse cette procédure. La décision de l'Assemblée plénière de la Chambre pénale de l'Audience nationale indique, dans son ordonnance du 2 décembre, qu'elle déclare «le manque de compétence objective » du Tribunal central numéro cinq dans cette procédure. Elle laisse ainsi sans effet tous les actes et résolutions postérieurs, y compris l'ordonnance de dessaisissement. Tous ces éléments ont abouti à l'arrêt momentané d'un processus dont les conséquences - d'un point de vue politique, juridique et symbolique - sont encore difficiles à mesurer. À l'heure où nous terminons ces lignes, le 26 décembre 2008, Garzón, en accord avec son ordonnance de dessaisissement, a envoyé une résolution avec des informations sur des « enfants perdus » aux doyens des juges d'instruction de Burgos, Barcelone, Madrid, Malaga, Valence, Biscaye et Saragosse. Il les invite à évaluer leur compétence dans l'instruction de ces cas.

Suite à cela, les associations qui se sont portées partie civile dans la procédure montraient des signes de fatigue. En effet, elles ont été très sollicitées tout au long de ce processus qui s'est déroulé avec un très faible soutien institutionnel et qui a demandé des moyens importants pour mener à bien, avec toutes les garanties, une enquête de cette amplitude. Les plaintes face au manque de volonté et de soutien ont été permanentes. Le 29 novembre 2008, s'y est ajoutée la dissolution de la Plateforme de victimes de disparitions forcées du franquisme (PVDFF), dans laquelle étaient représentées les associations s'étant portées partie civile dans la procédure del'Audience nationale. Le jour même est créée la nouvelle Coordination de collectifs de victimes du franquisme (CCFV) en réponse à une controverse au sein des collectifs de plaignants. Les différends entre collectifs se centraient sur la gestion du capital symbolique acquis par le mouvement associatif avec la déclaration de compétence de Garzón. Parmi ces désaccords, on peut évoquer deux points : d'un côté, la représentativité de certains porte-parole devant les médias, de l'autre, la composition de la commission d'experts - qui n'a existé que pendant quelques jours - demandée par Garzón. Pour justifier la refondation, la nouvelle Plateforme parle d'un désaccord plus grand : la satisfaction de certaines associations et la déception d'autres (réunies dans la CCVF) par l'intervention globale de Garzón, notamment sa décision de se dessaisir du dossier ${ }^{11}$. Cette nouvelle situation augure des stratégies différentes et diversifiées de la part des diverses associations sur le front judiciaire. Pour celles-ci, le front est ouvert et même

(10) Ordonnance du 18-11-08, p. 49-75. Garzón fonde son argumentation sur la recherche de Ricard Vinyes, Irredentas: Las presas políticas y sus hijos en las cárceles franquistas, Madrid, Temas de Hoy, 2002, et celle de Ricard Vinyes, Montse Armengou y Ricard Belis, Los niños perdidos del franquismo, Barcelona, Plaza y Janés, 2002, sur les femmes emprisonnées et les enfants perdus.

(11) Cf. http://memoriadesaparecidos.blogspot.com/2008/12/comunicado-de-disolucin-de-la.html 
inévitable, malgré le contretemps qu’a signifié le dessaisissement du juge et, par la suite, la déclaration d'incompétence du Tribunal numéro cinq de l'Audience nationale pour juger les crimes du franquisme. On trouve parmi ces stratégies : le renvoi des cas vers les tribunaux territoriaux, suivant ainsi l'orientation donnée par l'ordonnance de dessaisissement de Garzón, le recours au Tribunal européen des droits de l'homme de Strasbourg ou l'élaboration d'une ligne de travail avec le Groupe de travail sur les disparitions forcées et involontaires de la Commission des droits de l'homme de l'ONU ${ }^{12}$. Alors qu'ils épuisent les instances judiciaires nationales, les collectifs civiques de récupération de la mémoire historique des victimes de la répression de l'arrière-garde franquiste - qui ont progressivement enrichi leurs requêtes de demandes sociales, politiques, économiques et juridiques -, semblent s'orienter vers des logiques transnationales de justice. Dans l'attente de nouvelles sur les différents fronts ouverts, le travail se poursuit sur le terrain.

\section{FOSSES DISPARUES}

Revenons à Villanueva de la Vera, pour illustrer comment est appréhendée et gérée, d'un point de vue local, la topographie de la répression franquiste, dont l'efficacité est en partie toujours d'actualité. Comme nous l'avons vu, il s'agissait de la première exhumation après la décision judiciaire de Pedraz autorisant de nouvelles excavations, alors que la compétence du juge Garzón pour enquêter sur les crimes du franquisme devait encore être définie. Par ailleurs, il s'agissait d'une période judiciaire très agitée, avec des nouveautés presque quotidiennes, des déclarations politiques grandiloquentes - ou, dans le cas du gouvernement, un silence quasi religieux - et une importante agitation médiatique. Ainsi, les excavations de Villanueva commençaient dans une atmosphère chargée, mais elles étaient aussi une initiative émouvante pour les associations qui promeuvent les exhumations en Espagne, notamment face à l'absurdité, à la cruauté et au caractère clairement « infrahumain » de la démarche. On cherchait, dans deux fosses séparées à moins de deux cents mètres de distance, cinq femmes exécutées par dix phalangistes locaux le 26 septembre 1936. Dans la zone, des prospections avaient eu lieu avec un géoradar (GPR de $400 \mathrm{MHz}$ ) et elles avaient eu pour résultat, publié dans un rapport technique, la détection et la localisation dans ces deux zones d'« anomalies électromagnétiques claires qui pouvaient être associées à des enterrements clandestins dans une fosse d'une profondeur estimée de $1,50 \mathrm{~m}^{13}$.»

(12) Cette voie a déjà été empruntée en 2002 par l'ARMH. Cf. Emilio Silva, Las fosas de Franco, Madrid, Temas de Hoy, 2005, p. 80-82.

(13) La prospection a été demandée par la Coordinadora de la Memoria Histórica de Extremadura (Coordination pour la mémoire historique d'Estrémadure). Le rapport a été rédigé en avril 2008. Les prospections avec un géoradar pour la localisation des fosses communes se font ponctuellement depuis 2002, mais elles se sont incorporées de façon plus systématique au processus d'exhumations, à travers Cóndor Georadar S.L., depuis la fin de l'année 2006. C'est à cette époque que le géoradar a commencé à être utilisé pour réduire la zone d'exhumation d'une fosse qui contenait quatorze corps à Fuenteguinaldo (Salamanca), et pour la recherche de fosses près des murs du cimetière de Merida. Je remercie Emilio Silva et Bonifacio Sanchez Cepa pour avoir éclairci certains points à ce sujet. 
L'excavation, organisée à Villanueva de la Vera par Lucio García Tornero, membre de l'association Nuestra Memoria, était liée à celle de deux autres fosses. Ces dernières ont été exhumées quelques semaines plus tard à Valverde de la Vera. Parmi les huit hommes trouvés, certains étaient les maris des femmes enterrées à Villanueva. Lucio avait de fortes raisons de s'impliquer dans ces tâches nécessaires, toujours compliquées et souvent frustrantes, réalisées pour mettre en place des exhumations. Elles impliquent non seulement d'obtenir des accords et des permis, de négocier avec les autorités, d'attirer une des équipes techniques disponibles ou de s'occuper de la presse, mais aussi, d'une façon très particulière, de se charger de la gestion des répercussions sur la municipalité, surtout lorsqu'il s'agit d'un cadre rural. À Las Albarizas, dans une des deux fosses des femmes, sa grand-mère et les deux sœurs de sa mère étaient enterrées, une des deux était enceinte et était mère d'un bébé de quinze mois. À Valverde, dans les deux fosses des hommes, il y avait un frère, un cousin germain et un beau-frère de sa mère. Parmi les treize personnes fusillées recherchées dans les deux localités, huit hommes et cinq femmes, six faisaient partie de sa famille. Selon ses propres mots, son effort n'avait pour objectif que de « réaliser le plus grand rêve de [sa] mère », pour qui le fait de savoir qu'une partie de la famille était « jetée comme des chiens dans les champs » était très douloureux.

Le cas de Lucio - réaliser le rêve de sa mère - est paradigmatique de l'expérience de nombreux proches qui ont maintenu discrètement, parfois secrètement et très souvent dans la peur, des attentes de sauvetage de leurs proches depuis de nombreuses années. Maintenant, dans le contexte des faits de la dernière décennie, ils ont pu petit à petit sortir du placard ${ }^{14}$. « Toute sa vie », Lucio a souhaité éclaircir ce crime et a petit à petit collecté des détails sur les faits qui se sont produits ces jours-là, surtout grâce à la « fraternité » semi-clandestine qui existait au sein des familles de victimes. Dans tous les cas, « avant, les gens parlaient peu. » Lucio n'avait que peu d'informations sur les exhumations de proches qui avaient eu lieu à la fin des années 1970. Après la tentative de coup de force de Tejero le 23 février 1981, elles ont été arrêtées. "Quand il y a eu Tejero, tout m'a paru sombre. Les gens ne voulaient plus parler, ce n'était plus possible, il y avait même encore plus de peur que lorsque Franco, lui-même, vivait. » Vers 1988, lorsque la peur d'un recul politique a diminué, il s'est à nouveau intéressé à la question, notamment grâce à son travail avec l'historien Julián Chaves, qui faisait alors des recherches sur la répression dans la province de Cáceres pour sa thèse de doctorat. Toutefois, ce n'est que lorsqu'il s'est convaincu que la Loi sur la mémoire historique avançait et qu'elle octroyait une couverture légale à des initiatives comme la sienne, que Lucio a envisagé de récupérer les restes de ses proches. À cette époque, à travers son ami Luis Ragel, il est entré en contact avec Emilio Silva (Président de

(14) Il ne s'agit pas d'une expression rhétorique. Nous l'avons entendue à de nombreuses reprises de la bouche de proches de victimes de la répression pour faire référence, comme dans le cas de l'homosexualité, au dépassement public et bien connu d'une situation antérieure de semi-clandestinité et même de honte, dans ce cas le fait d'appartenir à des familles de tradition rouge punies par la répression. 
l'ARMH) et l'exhumation a commencé à être planifiée. Les personnes qui étaient alors recherchées entre Villanueva et Valverde étaient, selon Lucio, « tous des disparus », des victimes de la période que l'historien Julian Casanova nomme «terror caliente» (terreur chaude), des premiers mois du conflit, fusillées par l'arrière-garde franquiste, sans procès dont parle l'ordonnance de Garzón. Malgré son enquête, menée pendant des années, Lucio n'avait réussi à trouver aucun document sur ces cas. Tout ce qu'on savait sur ce qui s'était supposément passé à Villanueva provenait de récits oraux partagés dans des cadres familiaux, entre familles de victimes et, plus récemment, dans la rue et d'autres cadres publics du village. Plus l'exhumation se rapprochait, plus elle suscitait des attentes diverses, de l'indifférence au rejet, en passant par l'engagement inconditionnel.

Après l'énumération du nom des cinq femmes, leur âge, condition, métier et descendance, voici le contenu d'un texte rédigé par Lucio, photocopié et distribué aux personnes présentes lors de l'exhumation de Villanueva :

Les circonstances de leur mort ont été reconstituées à partir de témoignages de proches et font partie de la mémoire historique, conservée dans cette localité, sur les faits qui se sont produits entre les mois de juillet et de septembre 1936. Au début du mois de septembre, plusieurs femmes sans défense ont été sorties de leur domicile à Villanueva par des phalangistes reconnus de la municipalité. Elles ont été détenues dans la mairie, utilisée à cette époque comme prison. On leur a rasé la tête, on les a humiliées en les obligeant à balayer les rues du village jusqu’à ce qu'elles soient finalement conduites à Las Albarizas, forcées à ramasser des haricots et fusillées par les tireurs qui les surveillaient.

«Elles ont été chassées comme des pigeons », disait-on dans l'entourage de l'exhumation, très influencé à cette occasion par la présence démesurée des médias. Selon Lucio - grâce à une des pirouettes du recyclage générationnel des mémoires familiales - cette expression était attribuée par une des nièces de Bernarda à sa grand-mère (la mère de Bernarda). Les circonstances précises de la violence de genre, sous-jacente à cette excavation, la coïncidence de cette dernière avec le moment fort de l'effet Garzón et son lien judiciaire direct avec l'Audience nationale en ont fait une fosse médiatique pour laquelle il y avait beaucoup d'attente ${ }^{15}$. À une époque de « limbes judiciaires », on cherchait énergiquement, sans résultats visibles, la trace du célèbre juge, sans savoir exactement où et comment la retrouver dans les protocoles d'exhumation, les décisions des techniciens responsables ou l'attitude et les

(15) À propos de l'impact des médias sur les exhumations et les débats entre les associations, cf. Francisco Ferrándiz, «La memoria de los vencidos de la guerra civil: El impacto de las exhumaciones de fosas en la España contemporánea », José Maria Valcuende y Susana Narotzky (eds.), Las políticas de la memoria en los sistemas democráticos: Poder, política y mercado, Sevilla, ASANA, 2005, p. 109-132. À propos du processus de « digitalisation » de la mémoire, cf. Francisco Ferrándiz y Alajandro Baer, « Digital Memory: The Visual Recording of Mass Grave Exhumations in Contemporary Spain », in Forum Qualitative Sozialforschung/Forum: Qualitative Social Research [Revista on-line], 9(3) : Art. 35, 2008. 
déclarations des familles. Le matin, dès que les travaux de localisation de la première fosse, coordonnés par Marisa Hoyos (ARMH) et l'archéologue Jimi Jiménez (Société de Sciences Aranzadi), ont commencé avec une pelleteuse, il y avait déjà plusieurs équipes professionnelles de tournage, parmi lesquelles se trouvaient plusieurs chaînes de télévision (dont deux étrangères) et deux équipes de documentaristes. L'émission « Esquina Viva » de la chaîne Estremadura Radio et plusieurs journalistes de quotidiens et d'agences d'information régionales et nationales étaient aussi présents ${ }^{16}$. Alors que la pelle travaillait, chaque instant suspect qui révélerait la présence d'une fosse était observé avec la plus grande attention et enregistré. On attendait une découverte matérielle concrète, idéalement un indice d'une fosse bien conservée, qui mettrait définitivement en relation le cas espagnol avec les flux de justice transnationale avec lesquels il commençait à converger. Néanmoins, il n'y eut que peu de «nouvelles » chocs. Cela ne signifie cependant pas que le processus d'exhumation et les cycles narratifs qui l'accompagnent n'ont pas été fondamentaux dans la négociation des mémoires de la répression franquiste à Villanueva, dans la région même de la Vera ou, comme cela se produit pour toutes les exhumations, dans le contexte national.

Les travaux de prospection se sont terminés à dix-sept heures, sans avoir trouvé autre chose qu'une couche de moins d'un demi-mètre de terre dense sur un lit phréatique. Cette situation était plus que décourageante pour un grand nombre de personnes présentes. Un membre du Forum pour la mémoire que j’ai rencontré pendant l'exhumation, Marco Rodríguez Peña, m’a emmené visiter le lieu d'une autre fosse dans la municipalité de Madrigal de la Vera, dernier village de la province de Cáceres sur la route qui mène à la province d'Ávila. Les itinéraires des activistes de la mémoire comme Lucio et Marcos se construisent par le biais de croisements permanents entre ce type d'endroits liés aux exécutions, des proches qui s'y rendent, les récits oraux plus ou moins embryonnaires ou structurés sur les faits et des fragments de documents de diverses origines - de documents d'archives à des lettres ou des photographies privées qui apparaissent dans le cadre d'une enquête. Une transposition extrême, dans ce qu'elle a de plus organisée et institutionnalisée, est constituée par les plans des fosses et les projets de récupération de tous les noms des personnes fusillées. Parmi ces documents, certains ont obtenu un soutien institutionnel et font partie des documents présentés au juge ${ }^{17}$. Les cas les moins documentés, comme celui de Madrigal, sont confrontés à une situation très différente de celle de la municipalité voisine de Villanueva.

(16) Parmi les chaînes de télévision se trouvaient ARTE, franco-allemande, et une chaîne canadienne. Dans l'après-midi, TVE nationale est arrivée. Parmi les journaux qui ont couvert l'information sur le terrain se trouvaient le Periódico de Extremadura, Hoy, Público et l'agence EFE. Pendant les jours qui ont suivi l'exhumation, des notes d'informations ont été publiées dans El Mundo (une page), ABC, La Razón, El País, 20 minutos et d'autres.

(17) Cf. par exemple, le projet « Todos los nombres de Andalucía » (Tous les noms d'Andalousie) (http://www. todoslosnombres.org/), ou celui de « Todos los nombres de Asturias » (Tous les noms des Asturies), dont la banque de données comporte 17700 noms, y compris ceux des exécutés et des morts au combat (http://www. todoslosnombres.es/). Sites internet consultés le 4 janvier 2009. 
Dans ce cas, il s'agissait d'une fosse présumée pour laquelle aucune enquête n'avait été menée, ni par des activistes locaux ni par des professionnels. On ignorait qui pouvait s'y trouver et il n'y avait apparemment aucun proche sur le point de réclamer une intervention. Toutefois, comme dans un grand nombre de lieux de la topographie de la répression franquiste, la fosse présumée n’avait pas perdu de visibilité dans le paysage. En effet, elle se trouvait à cheval entre la continuité, de plus en plus atténuée, de son efficacité terrorisante et une géographie de résistance ${ }^{18}$ semi-clandestine exprimée dans le respect d'une relative paix des morts, même s'ils sont anonymes et qu'ils ont été mal enterrés. Dans la partie basse d'un champ cultivé, il y avait un espace ovale couvert d'herbe non labourée. Il était ainsi depuis des décennies. «Un proche devrait remercier le propriétaire de l'exploitation, car il l'a toujours respecté, toute sa vie... », me disait Marcos. Sur place, il m’a raconté l'histoire suivante :

Une nuit, les chiens d'un berger qui était dans la zone ont commencé à aboyer, ils étaient très nerveux. Le berger, qui ne s'y trouvait pas, a pu entendre au loin des cris qui parlaient de clémence. « Ne me tuez pas, j’ai quatre enfants... » Ou je ne sais plus combien est-ce qu'il y en avait»,

histoire reconstruite par Marcos tel un ventriloque improbable. L'imploration et les hurlements des chiens dans la nuit sont ancrés dans la mémoire de la région comme le marqueur le plus évident qu'une exécution a eu lieu. Marcos ignorait qui pouvait être enterré dans la fosse présumée et seul un habitant du village avait signalé la possibilité que, selon ce que lui avait raconté son père à plusieurs occasions avant de mourir, son grand-père s’y trouverait. « C'est le seul. Pour le reste, tu parles avec les gens dans la rue et rien... Ici ce qu'il manque, c'est que quelqu'un dise : ...“c'est là que se trouve mon grand-père”. »

De retour sur les lieux de Las Albarizas del Tudal, presque plongé dans la nuit, des éléments nouveaux sont survenus dans la maison de campagne des proches d'Úrsula, où les personnes présentes à l'exhumation se sont abritées de la pluie intermittente. Le ton découragé avec lequel je les avais laissées quelques heures plus tôt était maintenant empreint d'un enthousiasme renouvelé. Bien sûr, disaient-elles, ils avaient effleuré la tombe avec la machine. Un vieux berger s'était approché de la zone et, en reconstruisant les témoignages que lui-même avait entendus tout au long de sa vie, il avait évoqué une nouvelle localisation de la fosse à seulement quelques mètres de l'aire prospectée. Les personnes qui étaient dans la maison sont descendues à nouveau dans le champ où se trouvait supposément la fosse pour parler du terrain avec les archéologues : évoquer les caractéristiques du nouvel emplacement, voir dans quelle mesure les témoignages collectés les mois précédents s'accordaient ou non et étudier la possibilité que les restes d'Úrsula et de Bernarda soient conservés, si elles avaient été enterrées en ce lieu.

(18) Steve Pile, «Introduction: Opposition, Political Identities and Spaces of Resistance », in Steve Pile y Michale Keith (dir.), Geographies of Resistance, London, Routledge, 1997, p. 2-32. 
Lucio a alors utilisé son pendule pour, en marchant plusieurs fois sur le lieu, essayer d'arracher un quelconque message d'espoir à l'endroit peu prometteur qui, à l'instar de l'ensemble du champ, avait une profondeur très proche du niveau phréatique.

Alors qu'on me racontait les nouveaux événements, la propriétaire du terrain, Charo, s'est présentée dans la maison. Elle a choisi ce moment pour partager avec les proches des femmes assassinées ce que la personne qui lui avait vendu le terrain vingt-huit ans auparavant lui avait dit : elle lui avait recommandé de ne pas remuer la terre qui se trouvait sous quatre jeunes bourgeons de chêne, si elle ne voulait pas trouver des restes humains de la guerre. À nouveau, le labourage avait été hésitant et il avait été arrêté près de la fosse présumée. Cet emplacement était juste de l'autre côté de la zone remuée par rapport au lieu signalé par le berger, mais cela multipliait le nombre d'options à explorer le lendemain. Nous sommes alors à nouveau descendus à l'endroit où la pelleteuse avait ouvert le terrain pendant toute la journée. Là, dans l'obscurité presque totale, Charo évoque la conversation qu'elle a eue avec l'ancien propriétaire de la ferme et montre ainsi qu'elle connaît bien les faits du 26 septembre 1936 qui circulaient de plus en plus dans l'entourage de l'excavation. Les récits, plus ou moins fugitifs, fragmentés ou consolidés qui survolent la recherche et, dans ce cas, l'ouverture des fosses, sont un élément essentiel dans l'exhumation et, comme nous le verrons par la suite, dans l'itinéraire qui permet de rendre sa dignité au souvenir des personnes exécutées ${ }^{19}$.

« Disons que tu veux labourer ce petit bout de terrain, ou y semer quelque chose, m’a-t-il dit, voilà le problème... » Il m’a prévenu et m’a dit de faire bien attention, que si je passais le tracteur, que je ne sois pas étonné car il y avait des femmes enterrées. Ensuite, on m’a raconté l'histoire, mon père et d'autres... Comment ils les avaient exécutées et tout...

Quelques semaines plus tard, Lucio complèterait la partie finale de l'histoire qui avait été nuancée et enrichie par les témoignages des descendants de certaines personnes liées aux faits et qui jusqu’à présent étaient restées en retrait. Les phalangistes avaient demandé à un groupe d'agriculteurs et de maçons qui étaient dans la zone d'enterrer les corps. Le chef de chantier d'un séchoir en construction dans la région leur a dit qu'une d'entre elles bougeait. « Donne-lui un coup sur la tête avec le dos de la houe », lui a-t-on ordonné, il a refusé. Deux des phalangistes l'ont alors achevée eux-mêmes. Le maçon, selon ce qu'a raconté son fils à Lucio quelques jours après l'exhumation, « n'a plus jamais été le même, il dormait avec une machette sous le coussin, il tremblait, il est tombé malade et est mort très vite. » L'exhumation avait révélé l'existence d'un mort de tristesse en relation avec l'exécution et ses proches y avaient eux aussi trouvé un espace pour raconter leur histoire. Le crachin continuait de tomber sur le champ de Las Albarizas. Comme cela s'était produit pendant toute

(19) Francisco Ferrándiz, «Cries and Whispers: Exhuming and Narrating Defeat in Spain Today », in Journal of Spanish Cultural Studies 9(2), 2008, p. 177-192. 
la journée, certains techniciens et activistes continuaient de recevoir des appels sur leurs téléphones portables demandant de l'aide pour localiser ou exhumer des restes dans la région, ou des informations pour compléter une chronique. Lucio marchait, pensif, sur le lieu, en long et en large. Son pendule commença à s'agiter, signalant une présence sous la terre, mais il émettait un message déconcertant dans son oscillation spasmodique. Ce n'était pas de l'eau, mais on ne pouvait pas non plus déterminer de quoi il s'agissait.

Le lendemain, après plusieurs cycles successifs d'espoir et de découragement, les possibilités liées aux deux autres endroits se sont épuisées, sans laisser aucune trace. Dans l'autre fosse, où se trouvaient la grand-mère et deux tantes de Lucio et que certains des proches ont eux-mêmes commencé à fouiller avec des houes, rien de nouveau n'apparut pendant la journée. La tentative de recherche des restes a donc été stoppée. La désolation face à l'absence de résultats concrets dans une exhumation qu'on estimait fondée sur du solide - tous les témoignages coöncidaient approximativement sur les localisations - était en partie compensée par l'effort, au moins, de les avoir cherchées après avoir épuisé toutes les possibilités : en creusant, en déplaçant des pierres et en aidant à passer au crible la terre quand cela était nécessaire pour ancrer les témoignages dans la localisation réelle de la fosse. Tous les proches présents ne partageaient pas le même avis sur l'intérêt d'une projection publique de l'excavation et encore moins sur la présence d'autant de médias. Toutefois, pour Lucio, le plus petit geste de l'excavation signifiait revendiquer avec force le souvenir dans l'espace public, même si ce n'était que pour quelques heures. On ne se limitait plus au noyau familial ou au village. À partir de ce moment, en Espagne et même ailleurs, les gens connaissaient la tragédie de ces cinq femmes. La requête a été notablement inscrite dans un document officiel de l'Audience nationale. Leurs restes, si les localisations évoquées étaient exactes, ont été dévorés par la terre.

Cependant, le cycle n'est pas fini : d'autres prospections sont envisagées dans la zone (prévues pour le printemps 2009) - fondées sur une nouvelle reconstruction en miroir de la localisation des faits, en partant du chemin et de la fontaine qui servaient de références pour les enterrements -, ainsi qu'une commémoration et le développement d'un site internet.

Un mois plus tard, les 28 et 29 novembre, l'équipe technique est revenue sur le site de «Las Palomas » dans la municipalité de Valverde de la Vera, pour chercher deux autres fosses dans lesquelles se trouvaient des voisins de Villanueva de la Vera exécutés eux aussi pendant la période nommée « terror caliente » de l'automne 1936. Sous un déluge, dans un terrain qui est rapidement devenu un grand bourbier, les fosses ont cette fois donné, au moins, une brève piste pour signaler que les séquences de terreur et de mort évoquées dans les souvenirs et les témoignages des familles et des témoins n'étaient pas une simple invention paranoïaque : à l'emplacement d'une des deux fosses recherchées, trois semelles de chaussure correspondant aux trois personnes exécutées recherchées - Teodoro Tornero, Lorenzo Cordero et Gregorio Recio - ont été retrouvées. À cela s'ajoutent un bouton rouge et une pièce de monnaie. 
Dans la fosse, il n'y avait rien d'autre, quelques rares objets personnels comme seules traces des treize personnes exécutées. Toutefois, bien que très peu de choses soient apparues, une partie importante du travail était fait. Ils étaient là. Il s'agissait de l'endroit où ils avaient été mal enterrés ${ }^{20}:$ « Jetés comme des chiens »

Il nous semble pertinent d'insister sur l'importance qu'ont acquise les fosses communes et le champ de forces qu'elles génèrent encore : non seulement dans les débats de la dernière décennie sur la mémoire de la guerre civile, mais aussi dans le besoin d'apporter une réponse de la part de diverses instances du pouvoir politique et judiciaire aux requêtes de certains secteurs de la société civile, les héritiers des vaincus de la guerre. Ces requêtes font progressivement partie du « sens commun » démocratique. Chaque pelleteuse qui tombe, chaque houe qui creuse, chaque truelle qui retire de la terre autour d'une fosse commune ou d'un emplacement présumé dans un coin d'Espagne interpellent des espaces et des temps distincts. Parallèlement, chacune de ces actions reconfigure de complexes processus sociaux, politiques, juridiques et symboliques, comme un caléidoscope en mouvement perpétuel, parfois sporadique, parfois plus serein. Elles mobilisent des récits locaux, des discours politiques, des paradigmes historiographiques et scientifiques, des produits médiatiques et des interventions ou recréations artistiques de nature très différente, parfois même opposée $e^{21}$.

Dans ce texte, nous sommes partis de la spectaculaire, bien que brève, intervention de Garzón pour arriver à une exhumation (dans ce cas sans découverte de restes), mais en fait le cheminement des événements est inverse. En effet, Garzón, comme auparavant le gouvernement de Zapatero, a dû réagir face à la pression exercée pendant les huit dernières années par les requêtes des associations représentant les descendants des vaincus. Cette pression est fondée sur des stratégies «par élévation »-qui amènent d'ailleurs les associations à toujours tenter d'être au-devant des institutions ${ }^{22}-$, et s'ancre dans l'accumulation progressive des exhumations, des corps récupérés, des cartes des fosses d'échelles différentes, des listes des personnes qui ont subi des représailles. Il s'agit d'un cycle commémoratif émergent qui met progressivement en relation des lieux de répression, des assassinats et des massacres et une présence inégale, mais constante, des débats sur la « mémoire historique » dans les médias, dans

(20) À propos de la responsabilité politique de l'archéologie de la guerre civile et sur le besoin d'interpréter les lieux d'excavation et les restes matériels trouvés dans le contexte de toute la gamme de sites archéologiques du conflit disponibles, cf. Alfredo González-Ruibal, « Making Things Public: Archaeologies of the Spanish Civil War », in Public Archaeology 6(4), 2007, p. 203-226. Selon González-Ruibal, la prolifération contemporaine de lieux archéologiques de la guerre civile contraste avec son « invisibilité sociale » pendant des décennies, à quelques exceptions près, ce qui a limité son potentiel didactique jusqu'à présent. Tout comme les fosses communes, les autres restes de la guerre sont dans les limbes légaux, en n'étant pas reconnus légalement comme « patrimoine archéologique » (qualification réservée aux vestiges de plus de cent ans), bien que des « interprétations généreuses » de cette norme aient existé (ibid, p. 206-207).

(21) Francisco Ferrándiz, «Cries and Whispers: Exhuming and Narrating Defeat in Spain Today », op. cit.

(22) Je remercie Emilio Silva d'avoir souligné ce point. 
les institutions académiques - bien que dans une moindre mesure - et de manière significative au sein des conversations plus ou moins privées de nombreux citoyens dans des contextes divers ${ }^{23}$. C'est-à-dire, pour résumer, bien que ce soit avec un intérêt fluctuant, le débat semble bien ancré dans le tissu social dans lequel les positions sont multiples - c'est là aussi évident -, plus ou moins nuancées, et semblent parfois même irréconciliables.

Des exhumations de fosses communes comme celles de Villanueva et Valverde font partie d'une complexe et nécessairement controversée relecture contemporaine de faits tragiques dérivés d'une politique de répression systématique menée à bien par l'armée qui s'est soulevée, comme l'ont déjà signalé de nombreux historiens et comme cela a été écrit dans l'ordonnance de Garzón. Suite aux violences de l'arrière-garde des deux camps adverses, l'Espagne est devenue un paysage parsemé de fosses communes. Comme l'a signalé récemment Javier Rodrigo ${ }^{24}$, la reconnaissance d'abus graves dans les deux camps n'équivaut pas à leur symétrie. Dans son magnifique Hasta la raíz, il évoque cinq raisons pour lesquelles il est impossible, d'un point de vue historiographique, de défendre l'idée même d'une équivalence entre les répressions d'arrière-garde pendant la guerre civile. La première asymétrie est quantitative et elle fait référence aux nombres totaux. De plus, la violence putschiste a été proportionnellement plus importante dans son rapport au territoire contrôlé. Il y a aussi eu une plus grande violence de la part des troupes soulevées dans les territoires qui ont changé de camp dans les premiers mois de la guerre. Une autre asymétrie est liée aux dates pendant lesquelles la phase nommée «terror caliente » est devenue « represión fría » (répression froide). Enfin, plus la guerre avançait, plus l'arrière-garde des troupes soulevées était significativement plus importante que celle des Républicains. Cet auteur affirme d'ailleurs, au sujet du problème controversé des chiffres, que dans l'arrière-garde républicaine, on a évoqué le nombre d'environ 55000 morts, alors que dans celle des troupes soulevées, le nombre dépasserait largement les $100000^{25}$.

Après la fin de la guerre, la répression a utilisé, parmi ses multiples modalités, l'abandon méprisant et intentionnel des fosses communes des vaincus, devenues des instruments de la terreur permanente, des stratagèmes pervers d'une ténébreuse pédagogie du sang ${ }^{26}$. Dans une certaine mesure, ces éléments perdurent dans le présent,

(23) Cet aspect est aussi mis en avant par Fernández de Mata (Ignacio Fernández de Mata, « El surgimiento de la memoria histórica: Sentidos, malentendidos y disputas », in Luis Díaz Viana y Pedro Tomé (dir.), La tradición como reclamo, Salamanca, Junta de Castilla y León, 2007, p. 195-208). À propos du potentiel de mobilisation de l'arrivée des débats sur la mémoire historique dans les espaces d'expérience quotidienne, cf. l'incident raconté par Silva dans un récent entretien réalisé avec Jo Labanyi (Jo Labanyi, « Entrevista a Emilio Silva », p. 143-155, Journal of Spanish Cultural Studies 9(2), 2008, p. 148-149).

(24) Javier Rodrigo, Hasta la raíz: Violencia durante la Guerra Civil y la dictadura franquista, Madrid, Alianza, 2008.

(25) Dans sa résolution du 26 décembre 2008 sur les enfants perdus du franquisme, révélée le 7 janvier 2009 , Garzón précise que, selon ses expertises, le nombre total de victimes peut se situer entre 136062 et 152237.

(26) À propos de la « pédagogie du sang », cf. Javier Rodrigo, Hasta la raíz: Violencia durante la Guerra Civil y la dictadura franquista, op. cit., p. 73. 
même si leur efficacité diminue avec la mort progressive des générations qui les portent au plus profond d'elles-mêmes et pour lesquelles ils sont profondément ancrés comme paradigme de la répression. Il s'agissait d'un abandon sélectif qui prolongeait la vie utile des paysages de la peur et approfondissait le court-circuit du contrôle familial, social, politique et symbolique des morts et des deuils. Cependant, bien que le processus contemporain d'exhumations des fosses de la défaite soit sujet d'interrogation pour beaucoup, de stupeur pour d'autres, et a même été l'objet d'accusations de fanatisme - "guerracivilismo », anachronisme et même nécrophilie - l'Espagne est un pays qui a consacré de nombreux efforts à l'exhumation de la guerre civile depuis la fin du conflit. Nous entendons par là que les exhumations contemporaines ne sont pas une nouveauté et n'ont pas lieu dans le néant. En revanche, ce qui a effectivement changé de façon significative est le contexte dans lequel elles se produisent, leur portée et les conséquences qu'elles ont dans le réexamen de différents aspects de l'histoire du pays : depuis l'étude plus détaillée de la portée de la répression franquiste non seulement à court, mais aussi à long terme, jusqu’à la récente « tentative » de logiques transnationales de justice, ou le questionnement des possibles limites de la transition espagnole.

Depuis la victoire militaire, les morts du camp des vainqueurs, au front et à l'arrière-garde, ont été en bonne partie sujets d'enquêtes dans le cadre de la Causa General (Cause générale). Ils ont étélocalisés, déterrés, inhumés à nouveau et incorporés aux significatifs cycles commémoratifs et projets de monuments accompagnés d'une ardente rhétorique d'hérö̈sme et de $\operatorname{martyr}^{27}$. Malgré tout cela, la réactivation commémorative des fosses nationales dans le cadre du paradigme des tombés pour Dieu et pour l'Espagne n'est pas complète, car il manque la prise en compte, d'une part, de la façon dont certains groupes du camp des vainqueurs ont estimé être offensés par l'utilisation décharnée du régime de Franco des corps et de la mémoire de leurs proches, d'autre part, de l'offense subie avec les fosses républicaines. En effet, comme nous avons pu le voir, à la fin des années 1950 de nombreux cadavres ont été déplacés

(27) Cf. ABC, Arriba ou Alcázar dans les années d'après-guerre. On peut souvent y trouver des reportages sur des exhumations, des identifications et des «martyrs » enterrés à nouveau, aux côtés d'informations relatives aux enterrements publics, aux messes solennelles, à l'édification de monuments et à la pose de plaques commémoratives. À propos de leur importance significative, cf., par exemple, $A B C$ du 25 février 1940 qui se réfère à l'exhumation de 1500 « patriotes » dans le cimetière de Moncada à Barcelona. On peut aussi voir les ordres publiés dans le BOE (Bulletin Officiel de l'État) du 1er mai 1940, p. 3257, l'ordre « sur les exhumations de cadavres de personnes assassinées par les rouges », signé par Serrano Suñer (je remercie Carlos Agüero de m'avoir fourni cette information). Paradoxalement, comme le signalent des auteurs tels que Javier Rodrigo, la Causa General (Cause Générale), conçue comme la version officielle de la dénommée « terreur rouge » a eu pour conséquence imprévue à long terme l'inhibition de la recherche contemporaine sur les fosses franquistes et sur certains faits de la violence par l'arrière-garde républicaine. Ce débat continue de se dérouler dans les «mythes, anathèmes et justifications », plus que dans celui des recherches rigoureuses (Javier Rodrigo, Hasta la raíz: Violencia durante la Guerra Civil y la dictadura franquista, op. cit., p. 83). À propos de ce point en particulier et de l'importance de s'intéresser aux « fosses de la République », cf. aussi l'instant book de Ranzato, El pasado de bronce (Gabriele Ranzato, El pasado de bronce: La herencia de la guerra civil en la España democrática,

Barcelona, Destino, 2006, p. 97-112). À propos des politiques de la mémoire pendant le franquisme, cf. Paloma Aguilar, Políticas de la memoria y memorias de la política, Madrid, Alianza, 2008, p. 95-231. 
des fosses communes pour être ré-inhumé dans le pharaonique Valle de los Caídos. On sait aussi que certains de ces corps sont issus des fosses républicaines alors que les familles concernées ignoraient tout. Ils sont enterrés sous la protection d'une plaque qui stipule «Tombés pour Dieu et pour l'Espagne 1936-1939 RIP » et couronnés, comme tous les corps qui s'y trouvent, par les tombes, de José Antonio Primo de Rivera et Francisco Franco. Des historiens prestigieux tel que Julián Casanova ont exprimé récemment leur étonnement face au secret qui entoure encore actuellement le nombre et l'origine des corps transférés au Valle, tout particulièrement entre 1958 et 1959. En effet, dans son livre pionnier, Daniel Suerio ${ }^{28}$ calcule qu'il pourrait y avoir environ 20000 corps au début de l'année 1959. Quant à Queralt Solé ${ }^{29}$, il parle d'un nombre se situant entre 20000 et 30000 . Le nombre total pourrait être bien supérieur ${ }^{30}$. Dans tous les cas, l'importance numérique du mouvement de cadavres de la guerre civile vers ce monument est bouleversante.

Par la suite, pendant les premières années de la transition, des groupes de proches ont ouvert certaines des fosses républicaines avec un rare, voire aucun, soutien institutionnel ou technique, bien que ces initiatives aient diminué après le coup d'État de $1981^{31}$. Il faut encore faire de nombreuses recherches sur ces exhumations puisque, avec l'élargissement des travaux de récupération des corps dans l'Espagne contemporaine, souvent, de nouvelles données apparaissent et leur nombre et échelle semblent être plus importants que prévu ${ }^{32}$. Dans une initiative en partie liée aux victimes directes de la guerre civile, en septembre 1995, le ministère de la Défense a signé une convention avec la fondation allemande Volksbund. Cette dernière se charge de la recherche des disparus de la Seconde Guerre mondiale, dans le but d'exhumer et, dans certains cas de rapatrier, les cadavres des soldats de la Division Azul morts dans la bataille de Leningrad et qui sont enterrés dans plusieurs cimetières russes ${ }^{33}$. Cependant,

(28) Daniel Sueiro, El Valle de los Caídos: Los secretos de la cripta franquista, op. cit.

(29) Queralt Solé i Barjau, Els morts clandestins: Les Fosses comunes de la Guerra Civil a Catalunya (1936-1939). op. cit., p. 57-90.

(30) Cf. l'article d'opinion de Julián Casanova dans El País, 20-11-07, p. 33, indiquant que le nombre de corps susceptibles d'être enterrés sous la crypte serait de presque soixante-dix mille personnes. Dans le documentaire Franco: Operación caídos diffusé le 28 janvier 2009, évoqué en note 2, l'abbé du Valle de los Caídos en personne, le père Anselmo, déclare devant la caméra : « officiellement, selon les livres, il y aurait 33897 personnes. Mais, il pourrait y en avoir bien plus [...]. D'un autre côté, de manière à ne pas alarmer les gens au sujet de la quantité de morts qui sont arrivés au Valle, etc., le calcul a été presque systématiquement fait en dessous de la réalité officielle. » À la question, « oseriez-vous donner un nombre officieux des personnes qui pourraient être enterrées ici ? », il a répondu « Je vous cite celui qui... parmi ceux qui étaient chargés des opérations... il approcherait les 60000 personnes. » À propos de la complexité et des possibles ambiguïtés contenues dans ce monument, cf. Paloma Aguilar, Políticas de la memoria y memorias de la política, op. cit., p. 146-158. Selon cette auteure, «l'indice de réconciliation n'affecte que, de plus tardivement, certains morts du camp républicain, mais en aucun cas les survivants de ce dernier » (ibid., p. 156).

(31) Francisco Ferrándiz, «The Return of Civil War Ghosts: The Ethnography of Exhumations in Contemporary Spain », in Anthropology Today 22(3), 2006, p. 7-12.

(32) Certaines de ces exhumations ont bouleversé l'opinion publique nationale, tout particulièrement dans les pages de la revue Interviú.

(33) On calcule qu'environ 47000 soldats de la Division Azul ont servi. Le nombre total de morts se situe 
c'est suite à l'initiative d'Emilio Silva qui, en octobre 2000, a poussé à l'exhumation d'une fosse commune républicaine à Priaranza del Bierzo (León) dans laquelle se trouvaient treize corps, dont celui de son grand-père, qu'un nouveau chapitre dans la politique espagnole complexe de mémoire en relation avec la guerre civile a été ouvert. Cette exhumation s'est faite en présence de spécialistes (archéologues et médecins légistes) et a donné lieu à un rapport technique ${ }^{34}$. Depuis, environ deux cents fosses ont été ouvertes et plus de quatre mille corps ont été récupérés ${ }^{35}$. Le « travail de mémoire » s'est énormément diversifié, avec un parcours social, symbolique et politique très étendu et profond que même les plus visionnaires, au début, n’ont pu anticiper ${ }^{36}$. Dans ce processus, les fosses communes de la défaite ont évolué du statut de poubelles politiques, émotionnelles et symboliques, artefacts endommagés de la machine de terreur franquiste, à celui de puissants outils de mobilisation du débat public. Il est clair que l'impact médiatique de leur ouverture dans la société d'information et le fait qu'elle soit connue a été très important. Cependant, les dimensions que prennent les débats sur les politiques de la mémoire dans la société espagnole ne peuvent pas être uniquement le fruit de la projection médiatique et de la « consommation rapide » de restes humains, victimes de flagrants signes de violence et déterrés soixante-dix ans plus tard. Ils répondent surtout à un courant de fond, et non pas latent, auquel il suffisait d'une ouverture pour émerger.

Selon Robben ${ }^{37}$, la présence continue de fosses communes et collectives de personnes disparues peut injecter de grandes quantités de désordre, d'angoisse et de division dans toute société à court, moyen ou long terme. Dans le cas espagnol,

autour de 5 000. Dans une réponse écrite à une question d'Amparo Valcarce (Groupe socialiste) sur les travaux réalisés, dans le Boletín Oficial de las Cortes Generales (Bulletin officiel des deux Chambres) du 6 mars 2003, le gouvernement informe que le nombre total des exhumations réalisées depuis 1997 et jusqu'en 2001 était de 1162 cadavres de la Division Azul, déterrés dans quinze cimetières différents. L'intention était de réinhumer un bon nombre de ces derniers dans un secteur ad hoc rénové spécialement pour recevoir ces corps dans le cimetière allemand de Pankovka (Novgorod, Russie), cimetière de la 1 ère Division de la Luftwaffe. En réponse, on affirmait que 829 cadavres y avaient déjà été enterrés et qu'un budget pour un monolithe, des plaques avec les noms des personnes enterrées, des chemins d'accès et des sapins pour entourer le monument avait été prévu. Jusqu'à présent, la somme allouée pour une collection documentaire, une étude topographique et l'exhumation était de 90000 euros, estimant les frais pour le conditionnement du cimetière à plus ou moins $40000 €$. Cf. http://www.congreso.es/public oficiales/L7/CONG/BOCG/D/D 500.PDF\#page=311

(34) Emilio Silva, Las fosas de Franco, op. cit., p. 51-62.

(35) Les cas établis pour les fosses communes sont très différents et font référence à des fosses indépendantes ou à des fosses qui contiennent des centaines, voire des milliers de corps. Pour comprendre la progression, bien qu'il ne s'agisse pas de données officielles, il suffit d'évoquer deux cas. Dans le dossier sur les fosses communes, y compris le numéro 299 de la revue histoire et culture de L'Avenç (2005), on compte soixante fosses exhumées depuis l'année 2000, avec un total de 469 corps récupérés. En septembre 2008, dans un supplément dominical d'El País, en se fondant sur leurs propres données et d'autres apportées par l'ARMH, AGE-MHA, la Generalitat de Cataluña et la Junta de Andalucía, le journal comptabilise plus de 4000 corps exhumés dans 171 fosses depuis l'année 2000. Ces nombres, qui ne sont que des approximations, sont rapidement devenus obsolètes. Cf. El País, Domingo, 14-09-08, p. 4.

(36) Elizabeth Jelin, State Repression and the Labors of Memory, Mineápolis, University of Minnesota Press, 2003.

(37) Antonius Robben, « State Terror in the Netherworld: Disappearance and Reburial in Argentina », en J.A. Sluka (dir.), Death Squad: The Anthropology of State Terror, p. 91-113, Filadelfia, University of Pennsylvania Press, 2000, p. 93-97. 


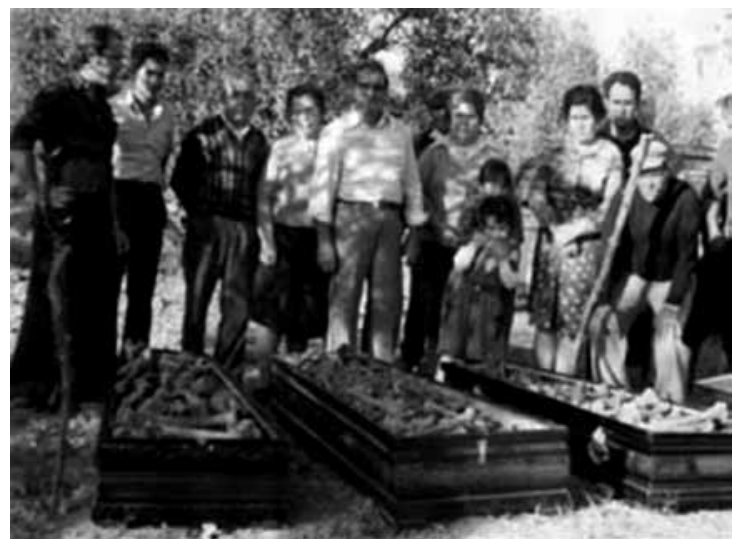

Pose collective suite à une exhumation menée à bien par des proches de personnes assassinées par le franquisme à Casas de Don Pedro (Badajoz) en 1978. L'absence d'aide technique dans ce cycle d'exhumations s'exprime dans l'accumulation des restes dans les cercueils ce qui ne permet pas de les différencier. Photographie prêtée par Emilio Silva.

cette situation se vérifie facilement : des paysages de terreur paralysants pendant les moments les plus durs de la répression, des distributeurs de peur pendant des décennies et, aujourd'hui, des éléments controversés. Avec le temps, les champs de méconnaissance, d'ambiguïté et de méfiance que génèrent les fosses communes de la guerre civile ont évolué restant des espaces de mort et devenant, également, des espaces « herméneutiques de suspicion et de révélation ${ }^{38} »-$ sculptés coup après coup, pelletée après pelletée, pour permettre la paralysie politique, sociale, économique et émotionnelle des adversaires pendant le conflit et, par la suite, des vaincus. D'une façon peut-être surprenante, elles sont aujourd'hui comme une blessure inattendue, puisque leur gestion a été reportée depuis des décennies. Le fait que la question des disparus et des exhumations soit arrivée jusqu’à Garzón, atteignant ainsi le niveau de figures juridiques relatives aux crimes contre l'humanité, est un exemple de leur efficacité toujours opérationnelle et du besoin de trouver une solution permanente. Cela montre aussi l'impossibilité de cacher le linge sale du conflit sous le tapis en attendant sa désactivation spontanée. D’un côté, leur caractère de secret public, le fait que ces fosses soient prisonnières du mépris des vainqueurs et du silence imposé aux victimes de cette violence, a empêché l'apparition non seulement de récits socialement légitimés sur la terreur qu'elles contiennent, mais aussi d'espaces de deuil consolidés. De l'autre, la répartition des fosses sur l'ensemble du territoire national cherche à construire une intentionnalité exemplaire exprimée dans une topographie dense de la terreur qui a permis à la machine de mort et de production massive de peur d'opérer à plein régime et à grande échelle.

La vague croissante d'indignation des proches de ces exécutions de l'arrière-garde se manifeste au travers des associations, des exhumations et des ré-inhumations et des actes commémoratifs de nature diverse. Ces manifestations se sont répandues dans la

(38) Michael Taussig, Shamanism, Colonialism and the Wild Man: A Study in Terror and Healing, Chicago, The University of Chicago Press, 1987, p. 10. 
société civile (non sans réponse) et ont réussi à faire pression sur des gouvernements autonomes et même sur le gouvernement national pour qu'ils octroient un financement et un soutien logistique ou développent une législation. Parmi les multiples éléments constitutifs de l'indignation des proches se trouve l'idée que la fosse commune est une forme d'enterrement « infrahumain » qui doit être corrigée pour pouvoir parler de schémas de réconciliation effectifs. Les discours contemporains des associations pour la récupération de la mémoire historique sont toujours en transformation. Ils recyclent des champs sémantiques, des concepts et des expressions de provenances différentes: en partant d'expressions populaires et jusqu'à des termes juridiques techniquement complexes $^{39}$. Leur évolution dans la dernière décennie est très intéressante pour n’importe quel spécialiste des politiques de la mémoire dans l'Espagne contemporaine. Dans ce contexte, il existe un aspect fondamental dans la justification de la pertinence des exhumations et, par la suite, de la gestion adéquate des restes, tout particulièrement d'un point de vue politique et symbolique. On peut d'ailleurs signaler que la gestion se fait dans le cadre d'une « chaîne de surveillance » qui, si elle n'est pas clairement établie et objet d'un consensus entre tous les acteurs sociaux impliqués, peut provoquer des malentendus et des disputes.

En effet, une des raisons de cet élan, qui amène à déterrer des personnes depuis quelques années, est la notion très répandue, parmi de nombreux proches et activistes de la mémoire, que la fosse commune est une forme dite d'enterrement « infrahumain » - créé intentionnellement comme telle par les assassins franquistes. Ce dernier se cristallise dans l'expression jetés comme des chiens, très souvent utilisée pour décrire la perception collective des fosses communes de la défaite et que nous avons entendue de la bouche de la mère de Lucio pour faire référence aux six membres de sa famille exécutés à Villanueva, parmi lesquels se trouvent sa mère, ses deux sœurs et son frère ${ }^{40}$. Cette notion - utilisée pour désigner un mauvais enterrement, un enterrement insuffisant, un enterrement offensant, un enterrement de châtiment, un enterrement destiné à exclure les exécutés de la communauté même des morts, sans leurs rites, sans leurs deuils, empilés, indifférenciés, déréglés, « jetés », encore plongés dans la violence qui les a tués, congelés jusqu'à aujourd'hui dans les râles de l'exécution est à la base de la mobilisation de nombreuses personnes qui cherchent les restes de leurs proches pour leur donner, dans cette logique aussi, un enterrement digne les différenciant de celui des chiens et des nuisibles. Lorsque j’ai demandé à Lucio à quoi sa mère faisait référence lorsqu'elle utilisait cette expression, il m’a répondu :

(39) À propos de l'apparition de la mémoire historique dans l'Espagne contemporaine depuis une perspective anthropologique, cf. Francisco Ferrándiz, « La memoria de los vencidos de la guerra civil: El impacto de las exhumaciones de fosas en la España contemporánea », op. cit. et Ignacio Fernández de Mata, « El surgimiento de la memoria histórica: Sentidos, malentendidos y disputas », op. cit.

(40) Il s'agit, sans aucun doute, d'une expression très populaire qu'on entend très souvent dans l'entourage des fosses. Cette expression contribue aussi à la controverse entre lan Gibson et les proches de García Lorca. Pour l'hispaniste, très partisan de l'exhumation du poète : « Lorca repose jeté dans un fossé

comme un chien. » Cf., par exemple, http://www.lukor.com/not-soc/cuestiones/portada/08091213.htm 


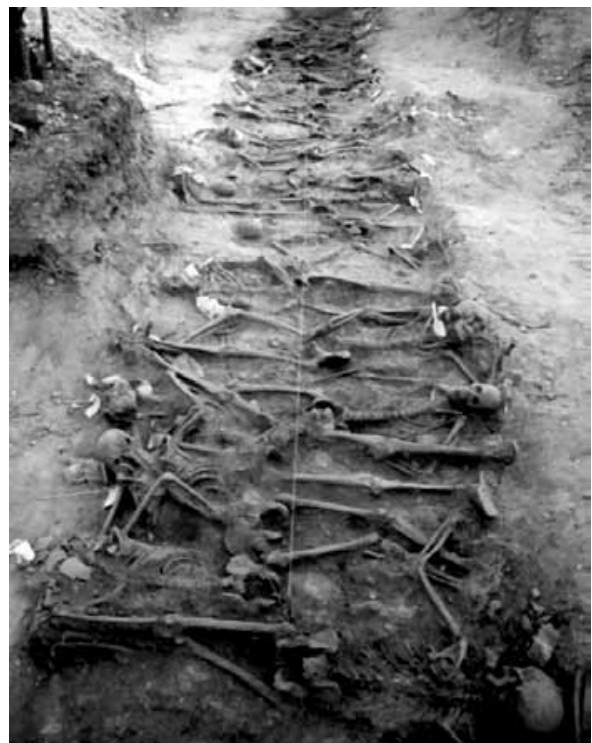

FIGURE 2: Restes des 47 personnes exhumées à Villamayor de los Montes (Burgos) en juillet 2004. La photographie est devenue une des images les plus emblématiques des fosses du franquisme. En 2005, elle a notamment été utilisée sur une affiche très choquante qui annonçait une conférence internationale à l'Université de Notre-Dame (Indiana). Elle a aussi figuré en couverture d'un des trois rapports publiés jusqu'à présent par Amnesty International à propos des exhumations en Espagne et intitulé « Espagne : en finir avec le silence et l'injustice. » Photographie prêtée par Francisco Etxeberria.

« elle sentait qu'ils n'étaient pas dans un cimetière comme les "personnes”, qu'ils ne lui avaient pas permis de les pleurer, qu'ils étaient là sans honneurs funèbres. » Dans ce sens, le processus d'exhumation et de réinhumation des restes serait un processus simultané de réhumanisation, en opposition avec le schéma systématique de répression, de discipline et de disposition intentionnellement offensive des cadavres dans des fosses sans nom ${ }^{41}$.

Le fait de rendre leur dignité aux corps tirés ou enterrés comme des chiens dans des caniveaux, des gouffres, des puits ou des fosses, fait partie d'une culture politique en expansion et en transformation qui a de nombreuses significations en fonction des collectifs de la mémoire dont il s'agit. Dans un texte précédent (2005), j'évoquais les controverses qui s'étaient produites les premières années entre diverses associations pour contredire le trait déshumanisant de la violence et de la répression franquiste, tel qu'il s'exprimait dans l'enterrement dans des fosses. Dans tous les cas, un processus de néoritualisation sans précédent était en marche. Face à l'absence de règles consolidées pour commémorer, ou exhumer et réinhumer des corps exhumés, les proches

(41) Javier Rodrigo, Hasta la raíz: Violencia durante la Guerra Civil y la dictadura franquista, op. cit., p. 62. Bien que l'analyse détaillée de la construction de la figure des rouges comme membres d'une anti-Espagne qui devait être anéantie sort des objectifs de cet article, il est intéressant d'évoquer à ce sujet des déclarations de Queipo de Llano à Radio Sevilla en 1936 reprises dans l’ordonnance de Garzón du 16 octobre 2008, à la page 12, citant comme source Francisco Espinosa (Francisco Espinosa, La justicia de Queipo, Madrid, Editorial Crítica, 2006). Queipo de Llano affirmait, pour justifier l'impunité et mettre en avant la condition sous-humaine de l'ennemi : « J'autorise à tuer, comme des chiens, n'importe quelle personne qui ose exercer une forme de violence sur vous : car si tel est le cas, vous seriez exempts de toute responsabilité. » Cf. aussi Javier Rodrigo, Hasta la raíz: Violencia durante la Guerra Civil y la dictadura franquista, op. cit., p. 62-64. 
ne pouvaient s'en remettre qu'à des cérémonies religieuses et à des rituels d'État franquistes. Les premières sont discutables pour beaucoup, mais satisfaisantes pour d'autres, quant aux seconds, ils sont, logiquement, inenvisageables. Pour certaines associations, comme celle qui veille sur la fosse d'Oviedo, la meilleure façon de rendre leur dignité aux morts impliquait la préservation de la fosse sans excavation, pour permettre de conserver le potentiel didactique de la barbarie dans le désordre et l'inhumanité propres à l'enterrement. Déterrer, dans cette logique, consisterait à effacer la page la plus explicite de la cruauté de la répression. Pour d'autres associations, pour rendre leur dignité aux morts, il fallait nécessairement exhumer dès que cela était possible et rendre les restes. Cela ouvrait alors un parcours post-mortem complexe et délicat qui prolonge ce que Verdery a caractérisé comme la vie politique des cadavres ${ }^{42}$. Ce parcours, qui a commencé avec les exécutions et l'enterrement dans les fosses communes (des deux camps), est perçu comme un instrument de la terreur politique. Verdery insiste sur deux éléments importants : d'un côté, déchiffrer le « type de temps qui se congèle ${ }^{43}$ » dans la disposition politique des corps ; de l'autre, clarifier le contexte historique à partir duquel on récupère et réinterprète les restes en question. Cependant, sur ce point, au-delà des convergences de base entre les associations proexhumations, des sensibilités en désaccord se sont consolidées. Elles concernent la façon de piloter cette nouvelle vie politique des corps exécutés des vaincus de la guerre civile qui, comme nous l'avons vu, constitue l'épisode le plus récent, commencé dès la fin de la guerre ${ }^{44}$, d'une séquence d'exhumations de fosses nationales et républicaines. Au sein de ces groupes, pour certains, les proches ont le pouvoir de générer et de recycler les circuits religieux, politiques, symboliques et émotionnels qu'ils considèrent opportuns pour exhumer et réinhumer les cadavres - y compris le très demandé même si parfois controversé transfert de la fosse à la sépulture chrétienne. Pour d'autres groupes, la politisation de tout le processus, selon des règles rituelles de la gauche comprenant, de plus en plus, des références républicaines, est un impératif pour rendre leur dignité aux morts, puisqu'il s'agit à l'origine de crimes politiques. Pour cette dernière sensibilité, l'intervention de l'Église serait tout à fait contre-productive étant donnée sa couverture idéologique de la guerre et du franquisme. Par ailleurs, l'Église refuse actuellement de soutenir le processus d'exhumation et soutient la béatification de martyrs, victimes de la répression républicaine ${ }^{45}$.

(42) Katherine Verdery, The Political Lives of Dead Bodies, New York, Columbia University Press, 1999.

(43) Ibid., p. 12.

(44) Certains intellectuels, activistes et spécialistes de la communication ont suggéré l'ouverture d'une nouvelle phase liée à la possibilité de rapatrier les corps d'Espagnols illustres morts en exil, bien que ce ne soit pour le moment que des gestes rhétoriques. Cf. par exemple la colonne d'opinion de Benjamin Prado « ¿Por qué no traer a España a Machado y Azaña? » (Pourquoi ne pas ramener Machado et Azaña en Espagne ?), publié dans El País le 19 novembre 2008. L'auteur défend l'importance symbolique et politique d'enterrer avec les honneurs ces hommes dans leur propre pays.

(45) Comme la récente béatification controversée de 498 « martyrs » de la guerre civile, le 28 octobre 2007 sur la place Saint-Pierre de Rome. Cet acte était destiné, selon les mots du pape Benoit XVI, à alimenter « la miséricorde, la réconciliation et la concorde pacifique ». 
Malgré des désaccords quant à l'aspect, au contenu et aux contextes d'énonciation et d'écoute, le récit des biographies des exécutés et des actes de violence dont ont souffert ces derniers, construits par ceux qu'on appelle parfois les donneurs de mémoire, contribue à rendre leur dignité aux morts et fait l'objet d'un certain consensus. Le récit et le registre des témoignages de proches et de témoins, comme dans le cas de Las Albarizas de Vilanueva de la Vera, sont devenus un axe central du répertoire d'action de « récupération de la mémoire ». Pour les associations, ces récits ont un effet doublement thérapeutique. D'un point de vue personnel, dans de nombreux cas, ils brisent des décennies de peur, de honte et d'humiliation. D'un point de vue social, en se transférant à la sphère publique et en devenant visibles et légitimes, ils auraient comme conséquence la reconnaissance du rôle historique des victimes de la répression franquiste dans ce qui serait un acte de justice historique et de reconnaissance collective de leur souffrance ${ }^{46}$. Bien que ces mémoires orales aient été remises en question par certains historiens ${ }^{47}$, leur abondance et leur demande croissante impliquent que tous les espaces de récit - dans ce cas ceux de la défaite - n’ont pas été épuisés dans la société espagnole. Cela contredit d'ailleurs le sentiment de saturation produit par l'accumulation de l'important travail historiographique, la multiplication des discours politiques, médiatiques et maintenant juridiques et l'industrie culturelle qui a émergé dans le pays ces dernières années ${ }^{48}$. Comme l'a récemment signalé Paloma Aguilar, la maturité démocratique présumée du pays est mise à l'épreuve dans sa capacité à absorber ou non tout débat, quel que soit son degré de complexité, sur les politiques de la mémoire de la guerre civile, en assumant sa pluralité et en y plaçant les requêtes et les aspirations qui bénéficient d'un soutien social significatif ${ }^{49}$. Dans ce contexte, ces récits contemporains de la défaite racontés dans le cadre des exhumations, ainsi que d'autres lieux de la mémoire et les commémorations, semblent pouvoir échapper à leur destin présumé de résidus discursifs de la transition et réclamer un rôle plus central dans les politiques de la mémoire de l'Espagne actuelle ${ }^{50}$. Toutefois, ils ne doivent pas être perçus comme des discours objectifs et vérifiables sur le passé, mais

\footnotetext{
(46) Luis Diaz me signale l'importance que peut avoir dans ce contexte la « possibilité de se raconter » pour reconstruire une « identité humaine ».

(47) Cf. par exemple la controverse entre Espinosa (Francisco Espinosa, « De saturaciones y olvidos: Reflexiones en torno a un pasado que no puede pasar »), Juliá (Santos Juliá, « De nuestras memorias y nuestras miserias ») et Ruiz Torres (Pedro Ruiz Torres, « Los discursos de la memoria histórica en España » et «De perplejidades y confusiones a propósito de nuestra memoria ») dans la revue électronique Revista de Historia Contemporánea Hispania Nova 7, 2007. http://hispanianova.rediris.es/7/dossier.htm

(48) Francisco Ferrándiz, «Cries and Whispers: Exhuming and Narrating Defeat in Spain Today », op. cit.

(49) Paloma Aguilar, « Los debates sobre la memoria histórica », in Claves de Razón Práctica, 172, 2007, p. 6468, p. 68.

(50) Dans son étude sur les exhumations au Guatemala, Sanford insiste sur l'importance d'étudier les processus de paix et de réconciliation du bas vers le haut (Victoria Sanford, Buried Secrets: Truth and Human Rights in Guatemala, New York, Palgrave MacMillan, 2003, p. 122). Elle encourage aussi les chercheurs à s'intéresser aux traces de violence dans la structure même du récit des témoignages. Ainsi, «l'héritage du traumatisme est imprégné dans le langage, comme il l'est dans le reste des structures de la culture » (ibid., p. 18). C'est aussi le cas en Espagne, dans ce que nous pourrions caractériser comme une inscription à long terme recréée à partir du présent.
} 
en tant que réseaux de mémoires inégales, fragmentaires et multiples. Ils doivent être déchiffrés dans leur complexité au sein d'un contexte présent à partir duquel on se souvient : une société de l'information saturée, avec des cycles d'attention courts, spasmodiques et de nombreuses opportunités pour mettre en scène la souffrance. Ces mémoires ne sont pas non plus des générateurs de formes de victimisation acritiques, homogènes ou caricaturales, mais plutôt des fondements indispensables d'un système démocratique plus profond qui peut les contenir sans les occulter, les ignorer sans les dénigrer, dans le contexte de réseaux multiples et même contradictoires de la guerre, en partant des plus intuitifs et en allant vers les plus scientifiques ${ }^{51}$.

Cependant, le concept qui suppose de rendre leur dignité aux morts, tout comme les autres facettes d'un processus très dynamique et qui adopte parfois un rythme vertigineux, se transforme aussi, s'enrichit et se diversifie. Actuellement, pour certains collectifs, rendre la dignité implique encore dans leur répertoire de nommer, raconter, identifier, creuser, enterrer à nouveau, commémorer et ériger des monolithes. Tous les collectifs ou noyaux familiaux, selon leur style, s'influencent mutuellement malgré les polémiques. De plus, ils sont aussi alimentés par de nouveaux éléments commémoratifs issus d'autres latitudes. Par exemple, les premiers portraits et photographies de personnes exécutées que les proches ressortaient, apportaient et partageaient avec les personnes présentes lors des exhumations ont évolué. Ces images ont un usage de plus en plus politique, parallèlement à la consolidation et à la popularisation de la catégorie de disparus pour parler des personnes exécutées. On est face à un recyclage local de ces images montrées par les familles et les amis qui, comme le précise Dorfman sont devenues une « image très répandue, voire épidémique, de la tragédie et de la résistance qui fait maintenant partie de notre imaginaire planétaire au même titre que les marques et logos commerciaux ubiquistes. » Pour Dorfman, l'usage public de ces images transgressives, d'un immense pouvoir iconographique dans les manifestations et les rassemblements, est la réponse la plus appropriée aux disparitions. Cela contredit non seulement les politiques qui rendent invisibles des victimes, mais entre aussi en relation avec les nouveaux médias de manière « très efficace et extrêmement poétique ${ }^{52}$. Dans ce croisement grandissant d'actes destinés

(51) Francisco Ferrándiz, « Cries and Whispers: Exhuming and Narrating Defeat in Spain Today », op. cit.

(52) Ariel Dorfman, « The Missing and Photography: The Uses and Misuses of Globalization », in J. Santino (ed.), Spontaneous Shrines and the Public Memorialization of Death, New York, Palgrave Macmillan, 2006, p. 255260, p. 256. Dans une lettre signée par José Maria Pedreño, la Federación Estatal de Foros por la Memoria (Fédération étatique des forums pour la mémoire) a appelé à manifester le 1er février 2008 devant les tribunaux de toute l'Espagne. Elle demandait aux manifestants d'apporter des pancartes sur lesquelles était écrit « nos proches et d'autres victimes du franquisme » sous la devise « Justice, Vérité et Réparation », pour dénoncer l'« incapacité complète des victimes du franquisme à se défendre » suite à la décision de la Chambre pénale de l'Audience nationale. On peut consulter la convocation sur le site http://www.foroporlamemoria. info/media/2004/379_1_Carta_movilizacion.pdf et des images des manifestations sur http://www. foroporlamemoria.info/noticia.php?id_noticia=5886. À propos de la controverse autour de l'utilisation du terme « disparus », cf. la note 3. À propos de la popularisation de ces iconographies transnationales dans le cadre de la digitalisation de la mémoire traumatique de la guerre civile, cf. Francisco Ferrándiz y Alajandro Baer, « Digital Memory: The Visual Recording of Mass Grave Exhumations in Contemporary Spain », op. cit. 
à rendre leur dignité aux vaincus de la guerre civile espagnole avec les modalités d'hommage, de souvenir et de réparation qui font maintenant partie d'un répertoire transnational pour un nombre croissant d'associations et de proches, ce processus a maintenant un objectif premier indéniable. Il s'agit d'obtenir la reconnaissance publique (et idéalement juridique, malgré les problèmes actuels de la procédure lancée par Garzón) que ces morts ont fait partie d'un plan systématique d'extermination et, de ce fait, en accord avec la législation internationale des droits de l'homme, qu'elles sont des crimes contre l'humanité. 